Hearing Research 155 (2001) 161-174

www.elsevier.com/locate/heares

\title{
Expression of mRNA encoding extracellular matrix glycoproteins SPARC and SC1 is temporally and spatially regulated in the developing cochlea of the rat inner ear
}

\author{
Andrea J. Mothe, Ian R. Brown * \\ Department of Zoology, University of Toronto at Scarborough, Toronto, ON, Canada MIC 1A4 \\ Received 20 September 2000; accepted 16 January 2001
}

\begin{abstract}
SPARC is a multifunctional extracellular matrix (ECM) glycoprotein that shares partial sequence homology with SC1/hevin. These ECM molecules exhibit calcium-binding properties and modulate cellular interactions. This study examines the expression of SC1 and SPARC mRNA in the developing cochlea of the rat inner ear prior to and after the onset of hearing. At all ages examined, SC1 mRNA is highly expressed in neurons of the spiral ganglion. In contrast, SPARC transcripts are not detected in the spiral ganglion but are enriched in the temporal bone and cartilaginous otic capsule surrounding the cochlea. Both SC1 and SPARC mRNA are expressed in connective tissue elements involved in maintaining ionic homeostasis of cochlear fluids. SC1 mRNA is localized to type III fibrocytes of the spiral ligament ( $\mathrm{slg}$ ) and marginal cells of the stria vascularis, while SPARC mRNA is apparent in the spiral limbus and type I fibrocytes of the slg. At postnatal day 10, SPARC mRNA shows a dramatic change in expression. High levels of SPARC transcripts are induced in Deiters cells (dc) of the organ of Corti. Interestingly, this induction of SPARC mRNA correlates with the onset of hearing. This suggests that SPARC may play a role in calcium regulation in de when functional maturation of the cochlea is attained and rapid changes in calcium levels are required. (C) 2001 Elsevier Science B.V. All rights reserved.
\end{abstract}

Key words: Extracellular matrix; Calcium-binding glycoprotein; In situ hybridization; Organ of Corti; Deiters cell; Spiral ganglion neuron

\section{Introduction}

Rapid progress has recently been made in the characterization of genes expressed in the mammalian cochlea, the inner ear structure responsible for processing auditory stimuli. Several genes encoding extracellular matrix (ECM) proteins such as collagens are highly expressed in the cochlea (Robertson et al., 1994). The ECM plays a critical role during developmental events in the cochlea such as morphogenesis, differentiation,

* Corresponding author. Tel.: +1 (416) 287 7413;

Fax: +1 (416) 287 7642; E-mail: ibrown@scar.utoronto.ca

Abbreviations: dc, Deiters cells; ger, greater epithelial ridge; ECM, extracellular matrix; ihc, inner hair cells; m, modiolus; nb, Nissl body; oc, organ of Corti; ohc, outer hair cells; rm, Reissner's membrane; sg, spiral ganglion; sgn, spiral ganglion neurons; sl, spiral limbus; slg, spiral ligament; sp, spiral prominence; sv, stria vascularis and sensory cell synaptogenesis, in addition to the functional maintenance of the cochlea (Woolf et al., 1992; Lopez et al., 1995; Cosgrove and Rodgers, 1997; Legan and Richardson, 1997; Whitlon et al., 1999). Mutations affecting matrix molecules cause inherited forms of inner ear disease (Petit, 1996; Corwin, 1998; Cremers, 1999; McGuirt et al., 1999; Steel and Bussoli, 1999). Analysis of human fetal cochlear cDNA libraries has shown that in addition to collagen, SPARC is one of the most highly expressed genes in the developing cochlea (Robertson et al., 1994; Jacob et al., 1997; Skvorak et al., 1999).

SPARC is a calcium-binding glycoprotein that influences cell growth and differentiation by interacting with a range of ECM components, certain metals and cytokines (Yan and Sage, 1999). SPARC expression is temporally and spatially regulated during development, and is associated with cell turnover, remodeling and repair 
processes. Previously, we have cloned and characterized SC1 which shares partial sequence identity to SPARC (Johnston et al., 1990). The related ECM glycoproteins, SPARC and SC1, are thought to play a regulatory role in modulating cellular interactions. Hevin, the human homolog of rat SC1, was also identified from expressed sequence tags (ESTs) generated and sequenced from a cochlear library (Skvorak et al., 1999). Therefore, we were interested in determining the localized mRNA expression patterns of these related ECM glycoproteins in the developing rat cochlea. Selection of the rat as an auditory model for gene expression studies provides certain advantages since functional maturation of the cochlea occurs during the postnatal period.

Using in situ hybridization, we have examined the distribution of mRNA prior to and at the onset of auditory function, as well as in the mature cochlea. This study shows that SC1 mRNA was strongly expressed in neurons of the spiral ganglion (sgn) throughout development and in specific cell types of the lateral wall of the cochlea involved in maintaining homeostasis of the endolymphatic fluid. In contrast, SPARC mRNA was enriched in the surrounding cartilaginous otic capsule and temporal bone throughout development. A dramatic change in SPARC expression was observed concomitant with the onset of hearing at postnatal day 10 (P10). Pronounced onset of SPARC mRNA expression was observed in both the supporting Deiters cells (dc) which surround the outer hair cells (ohc) that mediate the transduction of sound stimuli, and in connective tissue elements responsible for maintaining the endolymphatic potential. These results suggest that the related ECM molecules, SPARC and SC1, play important roles in the development and function of the rat cochlea.

\section{Materials and methods}

\subsection{Riboprobe synthesis}

A 295-bp EcoRI-HindIII fragment of the rat SC1 cDNA was subcloned in pGEM-3 (Johnston et al., 1990) and was used to generate ${ }^{35} \mathrm{~S}$-labeled riboprobes. Linearization with EcoRI or HindIII and subsequent in vitro transcription incorporating ${ }^{35} \mathrm{~S}$-labeled UTP with SP6 or T7 RNA polymerases produced antisense and sense $\mathrm{SC1}$ riboprobes, respectively. SPARC riboprobes were generated from a 450-bp fragment of mouse SPARC cDNA (Holland et al., 1987) in pGEM-3Z. Linearization with $X b a \mathrm{I}$ and transcription with SP6 produced the antisense probe, and the sense probe was obtained by linearization with Pst I and transcription with T7 RNA polymerase. To generate non-radioactive DIG probes, the SC1 and SPARC cDNA frag- ments were subcloned into the riboprobe vector pBS and digoxigenin-dUTP was incorporated in the transcription reaction as described by Roche Biochemicals.

\subsection{Tissue preparation}

Timed-pregnant Wistar rats were lethally injected with pentobarbital and embryos at gestational day 20 (E20) were removed and rinsed in cold $0.1 \mathrm{M}$ phosphate-buffered saline (PBS, pH 7.4). Whole embryos were fixed in PBS-buffered 4\% paraformaldehyde overnight at $4^{\circ} \mathrm{C}$ before equilibration in increasing concentrations of 5,10 , and $20 \%$ sucrose in $0.1 \mathrm{M}$ PBS $(\mathrm{pH}$ 7.4) and subsequent embedding in OCT compound (Tissue-Tek, Miles Laboratories). P1, P5, P10, P14, $\mathrm{P} 20$, and adult rats were deeply anesthetized with pentobarbital $(50 \mathrm{mg} / \mathrm{kg}$, i.p.), perfused intracardially with cold 0.1 M PBS ( $\mathrm{pH} 7.4$ ), followed by PBS-buffered 4\% paraformaldehyde. Rats were decapitated, skin and outer ear flaps were removed to expose the ear canal, and the tympanic membrane was punctured. Whole heads or tissue blocks containing the middle and inner ear were dissected from the skull. As the cartilaginous capsule surrounding the membranous inner ear shows progressive ossification after birth, all postnatal specimens were decalcified immediately after dissection in $8 \%$ EDTA containing $4 \%$ paraformaldehyde at $4^{\circ} \mathrm{C}$ for a period ranging from 3 days to 4 weeks, depending on the age of the specimen. After decalcification, the tissue was cryoprotected by immersion in ascending concentrations of sucrose as described above and embedded in OCT compound.

\subsection{Radioactive in situ hybridization}

Serial cryostat sections of $20 \mu \mathrm{m}$ were collected onto gelatin-coated slides ( $1 \%$ gelatin, $0.5 \%$ chromium potassium sulfate) and air-dried overnight. Sections were postfixed in $4 \%$ paraformaldehyde for $30 \mathrm{~min}$ and rinsed in $0.1 \mathrm{M}$ PBS prior to protease treatment. Sections were incubated in concentrations of $1.0-2.0 \mu \mathrm{g} / \mathrm{ml}$ of protease $\mathrm{K}$ at $37^{\circ} \mathrm{C}$ for $20 \mathrm{~min}$ in $100 \mathrm{mM}$ Tris, 50 mM EDTA (pH 7.5), followed by a 10 min wash in 0.1 $\mathrm{M}$ triethanolamine ( $\mathrm{pH} 8.0)$, and dehydration through an ethanol series containing $0.33 \mathrm{M}$ ammonium acetate. Tissue sections were prehybridized for $1 \mathrm{~h}$ at $42^{\circ} \mathrm{C}$ in a solution containing $50 \%$ formamide, $750 \mathrm{mM} \mathrm{NaCl}$, $5 \times$ Denhardt's $(0.1 \%$ Ficoll, $0.1 \%$ polyvinylpyrrolidone, $0.1 \%$ bovine serum albumin), $0.1 \%$ sodium dodecyl sulfate (SDS), $100 \mathrm{mM}$ dithiothreitol, and $50 \mathrm{mM}$ PIPES ( $\mathrm{pH}$ 7.6). Hybridization was carried out in the same solution containing $1.5 \times 10^{6} \mathrm{cpm}$ per section of ${ }^{35} \mathrm{~S}$-labeled antisense or sense riboprobes at $55^{\circ} \mathrm{C}$ for 14-18 h. After hybridization, slides were rinsed in TES buffer $(10 \mathrm{mM}$ Tris, $1 \mathrm{mM}$ EDTA, $500 \mathrm{mM}$ 
$\mathrm{NaCl}$, pH 8.0), followed by a $20 \mathrm{~min}$ incubation at $37^{\circ} \mathrm{C}$ in TES buffer containing $20 \mu \mathrm{g} / \mathrm{ml}$ RNase A. Slides were then washed in TES buffer containing $300 \mathrm{mM}$ $\beta$-mercaptoethanol $\left(\beta\right.$-ME) at $37^{\circ} \mathrm{C}$ for $1 \mathrm{~h}$ followed by a 1 -h wash at $70^{\circ} \mathrm{C}$ in $0.1 \times \mathrm{SSC}$ and $300 \mathrm{mM} \beta$ ME. After post-hybridization washes, sections were dehydrated through an ethanol series containing $0.33 \mathrm{M}$ ammonium acetate and air-dried. Slides were processed for autoradiography with Kodak NTB2 liquid emulsion, and exposed for $4-6$ weeks at $4^{\circ} \mathrm{C}$. Slides were developed in Kodak Dektol developer for $2.5 \mathrm{~min}$, fixed for $5 \mathrm{~min}$ in Kodak Rapid Fix, washed in running water for $15 \mathrm{~min}$, and air-dried overnight. Emulsioncoated sections were counterstained with $0.1 \%$ cresyl violet and coverslipped with 50:50 xylene/Permount. Sections were examined under both brightfield and darkfield illumination. Data representative of at least six animals per age are shown.

\subsection{Non-radioactive in situ hybridization}

Sections for non-radioactive in situ hybridization were processed as described previously (Mothe and Brown, 2000). Briefly, after protease treatment as described above, slides were washed for $10 \mathrm{~min}$ in $0.1 \mathrm{M}$ triethanolamine ( $\mathrm{pH} 8.0$ ), followed by $15 \mathrm{~min}$ in $0.1 \mathrm{M}$ PBS ( $\mathrm{pH}$ 7.4). Sections were then prehybridized in a solution consisting of $50 \%$ formamide, $0.1 \mathrm{M}$ Tris (pH 8.0), $5 \times$ Denhardt's solution, $0.1 \%$ SDS, $0.05 \%$ $N$-lauroylsarcosine, and $50 \mathrm{mM}$ PIPES (pH 7.6). Tissue sections were hybridized overnight at $55^{\circ} \mathrm{C}$ in the same solution containing 50-100 ng/section of DIG-labeled riboprobes. Following hybridization, slides were subjected to TES washes and RNase treatment as described above. Post-hybridization washes were carried out at 37 and $65^{\circ} \mathrm{C}$ for $1 \mathrm{~h}$ each in TES and $0.1 \times \mathrm{SSC}$, respectively. Slides were rinsed in PBT buffer $(0.1 \mathrm{M}$ PBS, $0.1 \%$ Triton X-100) and incubated with $20 \%$ fetal calf serum in PBT for $2 \mathrm{~h}$. Sections were incubated with anti-DIG antibody conjugated to alkaline phosphatase (Roche) overnight at $4^{\circ} \mathrm{C}$. Sections were then washed in PBT buffer, equilibriated in AP buffer $(100 \mathrm{mM}$ Tris, $100 \mathrm{mM} \mathrm{NaCl}, 50 \mathrm{mM} \mathrm{MgCl} 2,0.1 \%$ Tween-20) $\mathrm{pH} 9.5$, and endogenous alkaline phosphatase activity was blocked by incubation in AP buffer containing $5 \mathrm{mM}$ levamisole. Tissue sections were incubated in substrate solution (AP buffer containing $4.5 \mu \mathrm{l} / \mathrm{ml}$ NBT and 3.5 $\mu \mathrm{l} / \mathrm{ml}$ BCIP; Roche) for 5-10 h. Slides were then rinsed in a solution consisting of $100 \mathrm{mM}$ Tris, $100 \mathrm{mM} \mathrm{NaCl}$ (pH 8.0) and dehydrated and coverslipped as described above.

Specimens of different ages were batch processed using both sense and antisense probes in parallel so that parameters of tissue processing were kept constant. Additional negative controls were performed, such as omission of the riboprobe in the hybridization buffer and prolonged incubation of sections with RNase enzyme prior to hybridization, which resulted in elimination of the signal. Tissue sections were examined using a Zeiss Axiophot microscope and images were captured with a Sony digital CCD camera (Empix) using Northern Eclipse 5.0 imaging software. Data representative of three animals per age are shown. The rat experimental procedure utilized in this study was approved by the University of Toronto Animal Care Committee.

\section{Results}

\subsection{Distribution of SC1 and SPARC transcripts in the developing cochlea of the rat inner ear}

In situ hybridization was employed to investigate mRNA expression patterns of the related ECM glycoproteins SC1 and SPARC during development of the rat cochlea. To provide a reference for the morphological structure of the auditory apparatus, tissue sections were counterstained with cresyl violet. As shown in Fig. 1A,B, sectioning through the mid-modiolar plane revealed the repeated structure of turns of the cochlea.

At the earliest time point examined, embryonic day 20 (E20), SC1 mRNA was expressed at high levels in the sensory sgn which will eventually relay auditory information from the sensory hair cells in the organ of Corti (oc) to the cochlear nucleus in the brainstem (Fig. 1A,C, brightfield and darkfield microscopy, respectively). The mid-modiolar cross-sections show clusters of sgn associated with each cochlear turn. The expression pattern and intensity of signal was similar in each turn. Strong SC1 expression was also evident in non-sensory regions of the cochlea such as the greater epithelial ridge (ger) (Fig. 1A,C). This is a transient structure which undergoes regression in early postnatal development to allow formation of the spiral limbus (sl) and inner sulcus. As shown in Fig. 1A,C, lower levels of SC1 mRNA were apparent in the stria vascularis (sv), and in the central core of the cochlea, the modiolus (m). The sv plays an important role in generating the endolymphatic potential in the cochlear duct. The maintenance of this positive potential and high potassium levels are believed to be important features for hair cell function (Steel and Bussoli, 1999). Potassium carries part of the transducer current through the hair cells, and the gap junction network which encompasses the supporting cells of the oc and the sl and spiral ligament (slg). These structures appear to provide a route for potassium recycling (Kikuchi et al., 1995). In contrast to the SC1 expression pattern at E20, mRNA encoding the related ECM glycoprotein SPARC was not present 

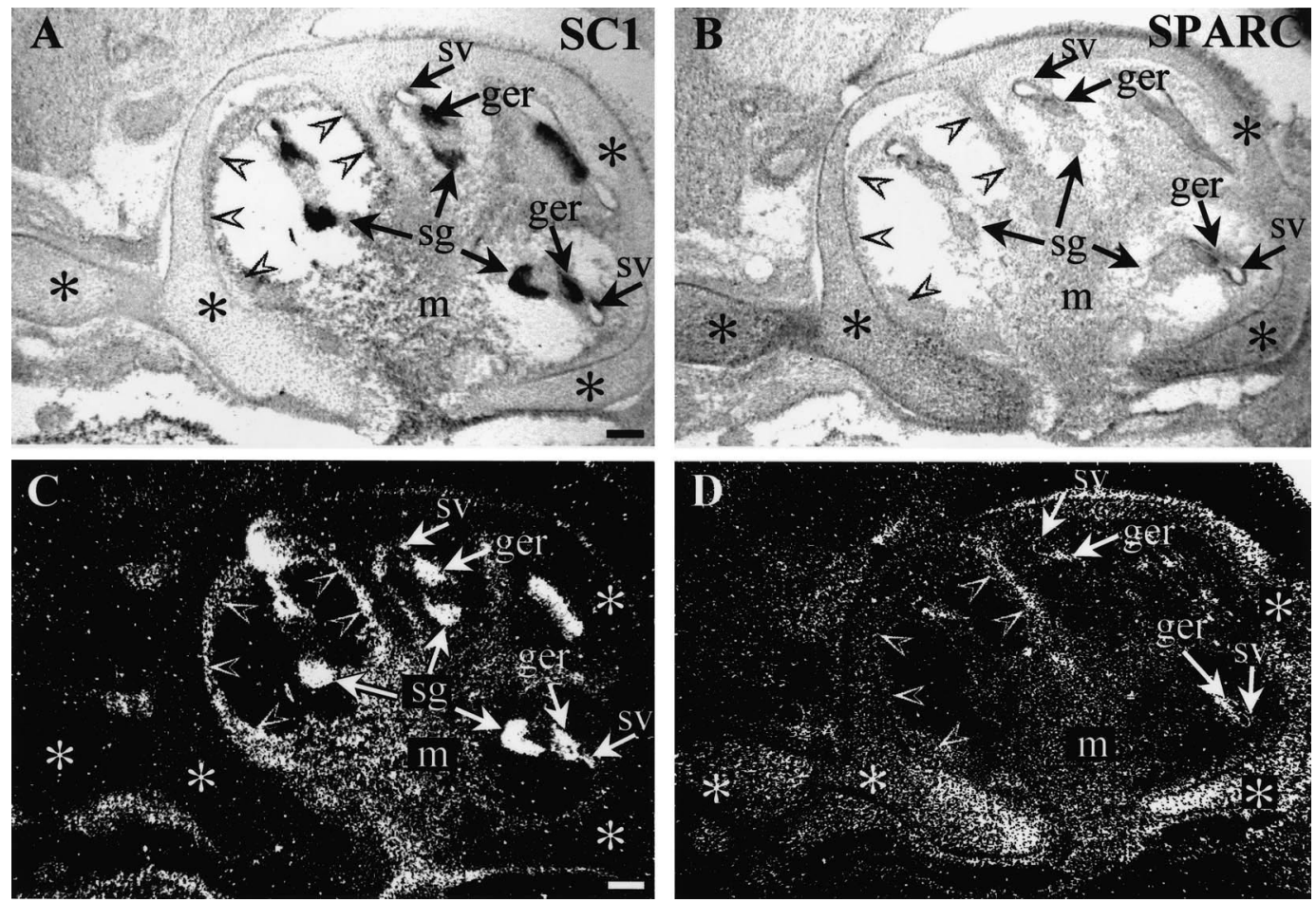

Fig. 1. In situ hybridization of SC1 and SPARC mRNA in the rat cochlea at embryonic day 20. (A) and (B) are mid-modiolar cross-sections of the cochlea hybridized with SC1 and SPARC riboprobes, respectively, and counterstained with cresyl violet to indicate tissue morphology as seen by brightfield microscopy. Corresponding darkfield images, shown in (C) and (D), indicate the distribution of silver grains representing $\mathrm{SC} 1$ and SPARC mRNA expression patterns. As shown in (C), SC1 mRNA is expressed at high levels in sn and in the ger. The high density of silver grains in these regions is seen as corresponding dark areas under brightfield illumination in (A). SC1 mRNA is also expressed in the $\mathrm{m}$ and lower levels of transcripts are evident in the sv of the cochlear duct $(\mathrm{C})$. In contrast, SPARC mRNA is enriched in the surrounding otic capsule indicated by the asterisks in (B) and (D), whereas SC1 mRNA is not apparent in this region (A and C). Both transcripts are expressed in the lining of the scala vestibuli and scala tympani, as indicated by the open arrowheads. Scale bar $=220 \mu \mathrm{m}$. ger, greater epithelial ridge; $\mathrm{m}$, modiolus; sg, spiral ganglion; sv, stria vascularis; asterisks, otic capsule; open arrowheads, lining of scala compartments.

in sensory areas of the cochlea such as the sgn. SPARC mRNA was apparent in the sv, ger, and $\mathrm{m}$ (Fig. 1B,D). In addition, SC1 and low levels of SPARC mRNA were localized to the epithelial lining of the scala compartments containing the perilymphatic fluid (open arrowheads in Fig. 1). This lining consists of simple unspecialized squamous epithelium of mesodermal origin. However, as indicated by the asterisks in Fig. 1B,D, SPARC mRNA was enriched in the cartilaginous otic capsule surrounding the membranous labyrinth, a region that did not express SC1 (Fig. 1A,C). The SC1 and SPARC riboprobes used in this study were specific and did not show cross-hybridization, as demonstrated previously by Northern blot analysis (Johnston et al., 1990). Adjacent sections hybridized with sense riboprobes showed no evidence of the specific hybridization obtained with the antisense probes (data not shown).

Fig. 2 highlights the differential expression pattern of SC1 and SPARC mRNA at P1 in the rat cochlea. At this stage, $\mathrm{SCl}$ mRNA was expressed at higher levels in the developing sv (Fig. 2C,E), compared to the weaker expression in this region at E20 (Fig. 1C). Similar to the expression pattern seen at E20, SC1 transcripts were enriched at P1 in the spiral ganglion (sg) and ger (Fig. 2A,C,E), while lower levels were apparent in the $\mathrm{m}$ (Fig. 2C). SPARC expression was enriched in the cartilaginous otic capsule (asterisks in Fig. 2B,D), whereas expression of SC1 mRNA was not detected in the otic capsule (Fig. 2A,C). SPARC expression was also evident in the sv and ger and, as shown at E20, SPARC mRNA was not apparent in the sg (Fig. $2 \mathrm{~B}, \mathrm{D}, \mathrm{F})$. In the lateral wall of the cochlear duct, higher levels of SC1 mRNA were associated with cells of the sv as compared to adjacent fibrocytes of the slg (inset in Fig. 2E), while the reverse pattern was observed for SPARC mRNA (inset in Fig. 2F). These regions and cell types of the cochlea possess an abundance of ion transport enzymes which function to maintain ionic homeostasis of the endolymphatic fluid. As shown by non-radioactive DIG probes at high magnification in Fig. 2G, SC1 mRNA was localized to the apical portion of the ger (ger ${ }^{\text {apical}}$ ). In sgn (in Fig. 2H), SC1 transcripts were distributed throughout the cytoplasm of the cell body but not into neuronal processes. At birth, the sg of the newborn rat consists of a homogeneous population of small, densely packed, spherical cells with 

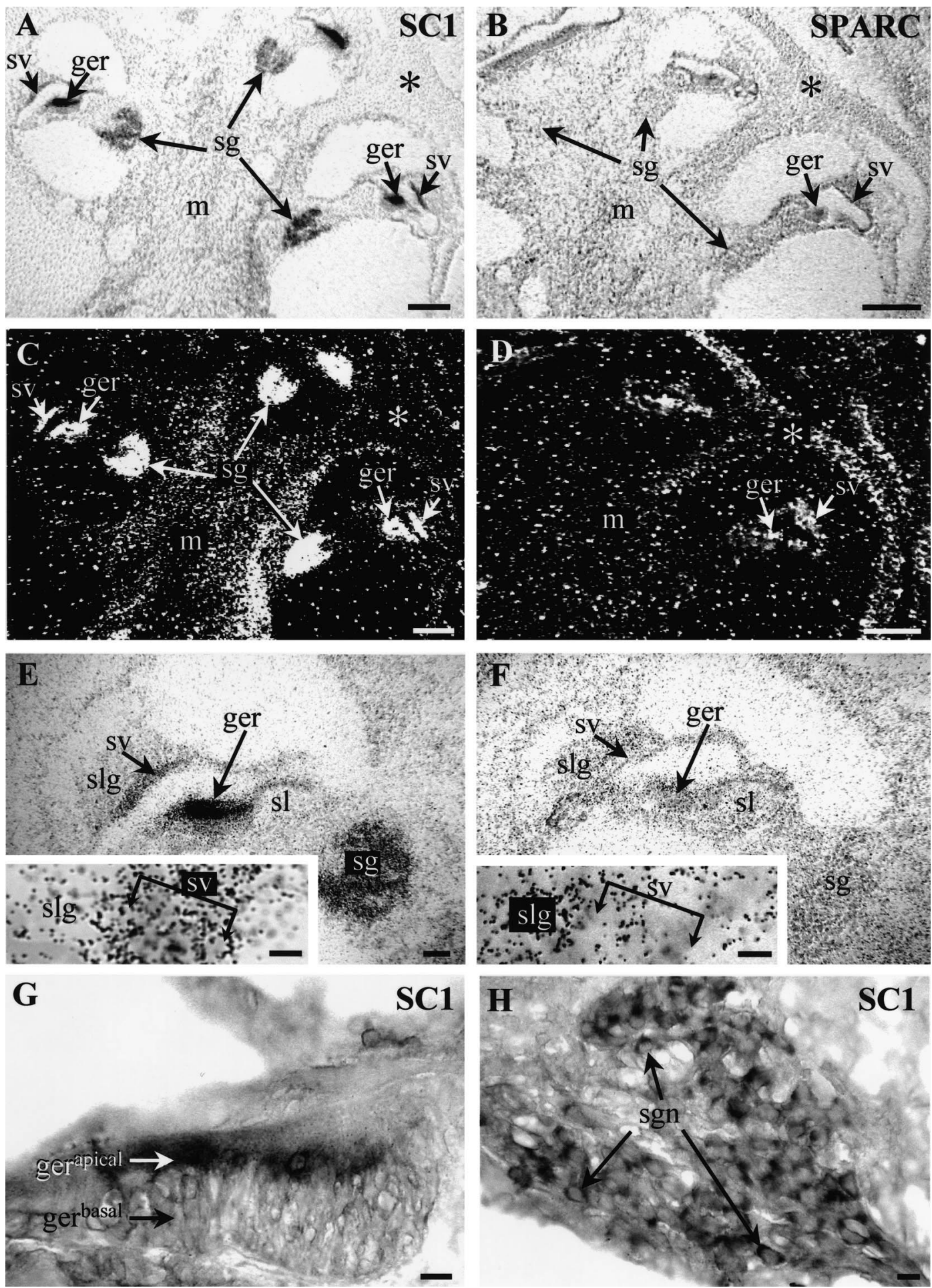

Fig. 2. Expression of SC1 and SPARC mRNA in the cochlea at P1. A strong SC1 mRNA signal is evident in the sg, ger, and the sv (A and $\mathrm{C}$, and at high magnification in E). In comparison, SPARC mRNA does not appear to be expressed by sn (B and D, and at high magnification in F). In contrast to SC1, weak SPARC expression is observed in the ger and sv (B and D). SPARC transcripts are enriched in the surrounding otic capsule as seen by the asterisks in (B) and (D). In contrast, SC1 expression is not apparent in the otic capsule (A and C). As shown at high magnification in the inset in (E), SC1 mRNA is enriched in the region of the sv, whereas lower levels of SPARC mRNA are apparent in the sv compared to the adjacent slg (inset in F). $(\mathrm{G})$ and $(\mathrm{H})$ are high magnification views of sections processed for in situ hybridization with non-radioactive DIG probes. SC1 mRNA is enriched in the apical portion of the ger (ger ${ }^{\text {apical }}$ in G), and in the cell body of sgn (in $\mathrm{H})$. Scale bar $=220 \mu \mathrm{m}$ in (A) $-(\mathrm{D})$; bar $=55 \mu \mathrm{m}$ in $(\mathrm{E})$ and $(\mathrm{F})$; bar $=10.9 \mu \mathrm{m}$ in insets of $(\mathrm{E})$ and $(\mathrm{F})$; bar $=17.3 \mu \mathrm{m}$ in $(\mathrm{G})$ and $(\mathrm{H})$. ger, greater epithelial ridge; ger ${ }^{\text {apical }}$, apical portion of ger; ger ${ }^{\text {basal }}$, basal portion of ger; m, modiolus; sg, spiral ganglion; sgn, neurons of the spiral ganglion; slg, spiral ligament; sv, stria vascularis; asterisks, otic capsule. 

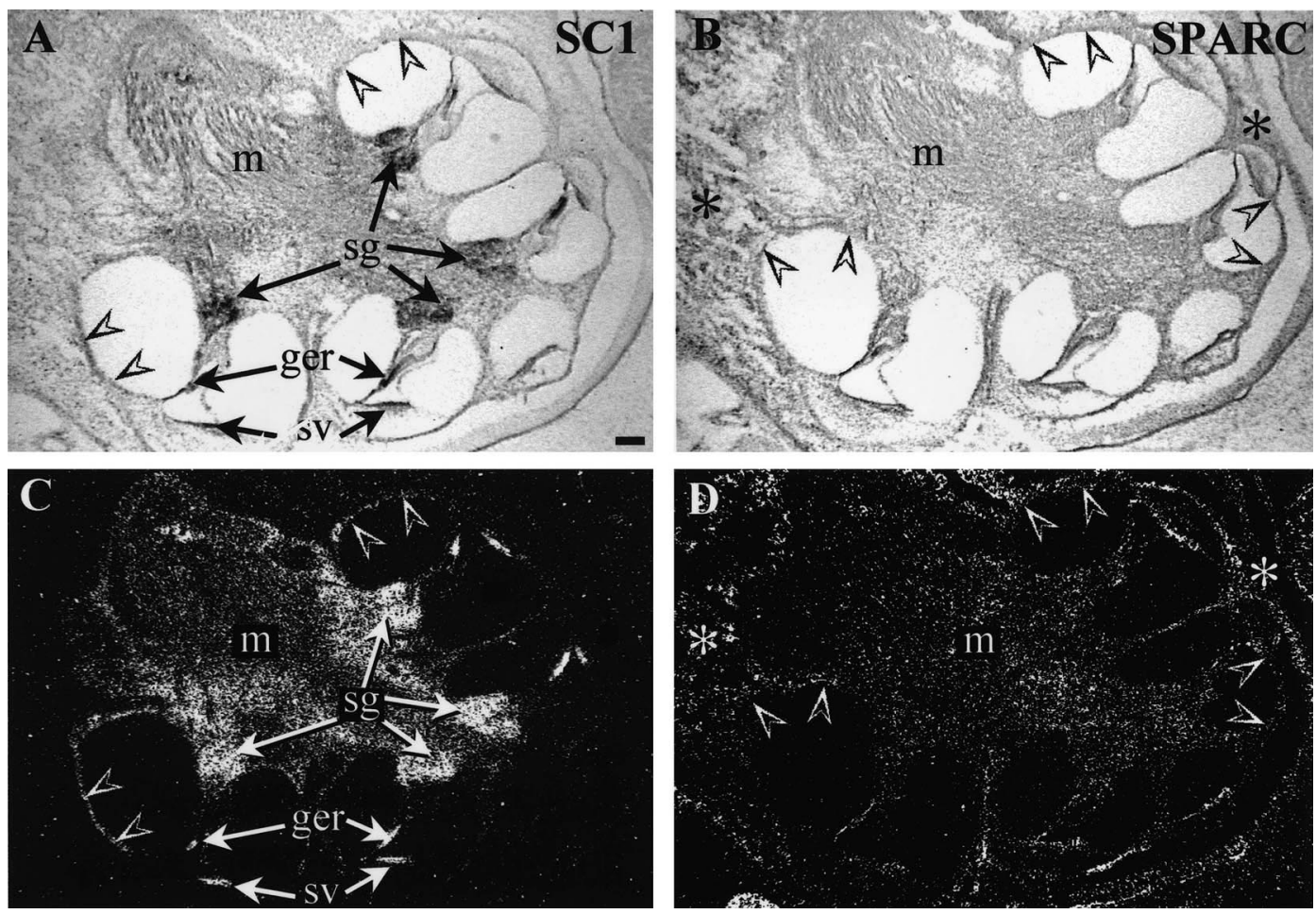

Fig. 3. Localization of SC1 transcripts in the rat cochlea at P5. As was seen at E20 and P1, SC1 mRNA continues to be strongly expressed at P5 in the sg, ger, and sv (A and C). Scale bar $=220 \mu \mathrm{m}$. ger, greater epithelial ridge; $\mathrm{m}$, modiolus; sg, spiral ganglion; sv, stria vascularis; asterisks, otic capsule; open arrowheads, lining of scala compartments.

large centric nuclei (Schwartz, 1986). This is reflected by the SC1 mRNA expression pattern in Fig. 2H. It is not until P8 that sgn acquire morphological features that are characteristic of mature sgn (Schwartz, 1986). As shown in Fig. 3, the pattern of SC1 and SPARC mRNA expression was maintained at P5 (Fig. 3A-D). Both transcripts were present in the epithelial lining of the scala compartments (open arrowheads in Fig. 3).

At P10, a striking change in mRNA expression patterns was observed. As shown in Fig. 4B, the expression of SPARC mRNA showed a sharp contrast to that observed at earlier ages in that high levels of SPARC transcripts were localized to discrete components of the cochlea. In particular, an intense signal was detected in the oc (Fig. 4B,D,F), the cochlear structure which contains the sensory hair cells and accessory supporting cells responsible for transduction of the auditory signal. In addition, high levels of SPARC mRNA were localized to the slg and sl, structures consisting of fibrocytes, cells which are rich in ion transport enzymes. SPARC mRNA was also enriched in the ossifying otic capsule indicated by asterisks in Fig. 4B,D. The strong SC1 mRNA signal observed in the ger at the earlier stages was not present (Fig. 4A,C) since by P10 the epithelial ridge has regressed. In addition to high levels of $\mathrm{SC} 1$ mRNA seen in the sg (Fig. 4A,C,E), SC1 transcripts were enriched in the sv and type III fibrocytes of the slg as shown by the white arrowheads in Fig. 4E. Lower levels of SC1 mRNA were associated with type I fibrocytes of the slg (Fig. 4E). In contrast, SPARC mRNA was higher in type I fibrocytes of the slg than the sv (Fig. 4F). Both SC1 and SPARC transcripts show very low levels of expression in the area of the spiral prominence (sp), consisting of type II fibrocytes.

\subsection{Specific localization of SPARC $m R N A$ in the organ of Corti at the onset of hearing}

As shown in Fig. 5B,C, the discrete localization of SPARC mRNA in the oc at P10 appeared to correlate with expression in dc, which are supporting cells that lie below the ohc. This was interesting since this developmental age correlates with the onset of hearing in rats. Non-radioactive in situ hybridization was performed to achieve a higher level of resolution. Representative sections were photographed under brightfield illumination to show the distribution of blue precipitate indicating SPARC mRNA, and phase-contrast microscopy was used to identify surrounding cell types in processed sections that were not counterstained. As shown in Fig. 5B under phase-contrast microscopy, SPARC mRNA was localized to the apical region of the elongated dc, whereas expression of SPARC in the sensory cells and other supporting cells was not apparent. The dc is elongated and forms a cup at its apex into which the basal pole of the ohc is inserted. A phalangeal process ex- 

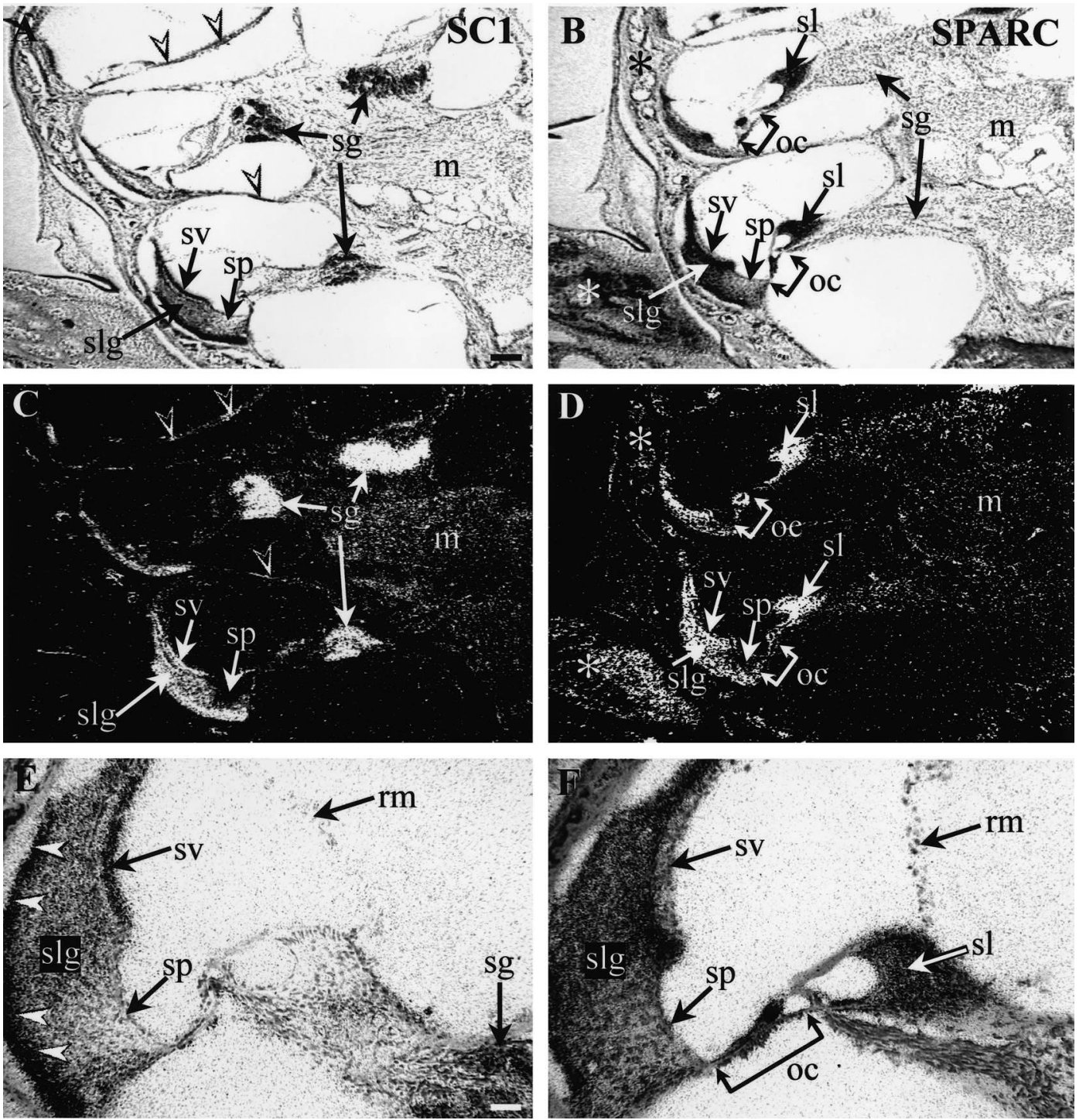

Fig. 4. Induction of SPARC mRNA in the oc at P10. A strong induction of SPARC mRNA is apparent at P10 in the region of the oc (B, D and F). SPARC transcripts are also expressed in the sl, slg (B, D, and F) and otic capsule (asterisks in B and D). Strong SPARC mRNA expression is evident in type I fibrocytes of the slg in relation to the weaker signal seen in the sv (F). In contrast, SC1 is strongly expressed in the sg and sv (A, C, and E). Higher levels of SC1 mRNA are evident in the sv and type III fibrocytes of the slg (white arrowheads) compared to type I fibrocytes of the slg (E). In addition, a diffuse distribution of SC1 mRNA is associated with the $\mathrm{m}$ and the lining of the scala compartments (open arrowheads) (A and C). Both transcripts show a weak signal in type II fibrocytes of the sp and neither transcript is expressed in Reissner's membrane (rm). Scale bar $=220 \mu \mathrm{m}$ for (A)-(D); bar $=55 \mu \mathrm{m}$ for (E) and (F). m, modiolus; oc, organ of Corti; rm, Reissner's membrane; sg, spiral ganglion; sl, spiral limbus; slg, spiral ligament; sp, spiral prominence; sv, stria vascularis; asterisks, otic capsule; open arrowheads, lining of scala compartments; white arrowheads, type III fibrocytes of slg.

tends from the dc body and inserts into the reticular lamina between ohc. In contrast to SPARC, SC1 mRNA was not detected in the oc (Fig. 5A). Fig. 5C shows a cross-section of $\mathrm{P} 10$ oc photographed under brightfield illumination to indicate the localization of SPARC mRNA to dc. An adjacent section in the same orientation was stained with cresyl violet to indicate cellular morphology of the oc for comparison (Fig. 5D). Based on the morphology of the oc, the major space between the nuclei of the dc and the basally positioned nuclei of the ohc consists of dc cytoplasm which forms the cup in which the ohc rest. It appears that SPARC mRNA is enriched in this cup region of the dc and is also apparent in the phalangeal process which extends up to the reticular lamina and surrounds the ohc (Fig. 5C).

As shown in Fig. 6, the distribution of $\mathrm{SC} 1$ and SPARC mRNA was examined in the cochlea at a later stage of development (P14) and striking differences were apparent. SPARC expression was enriched in the temporal bone (indicated by open arrows in Fig. 6B), compared to the absence of $\mathrm{SC} 1$ expression in these 

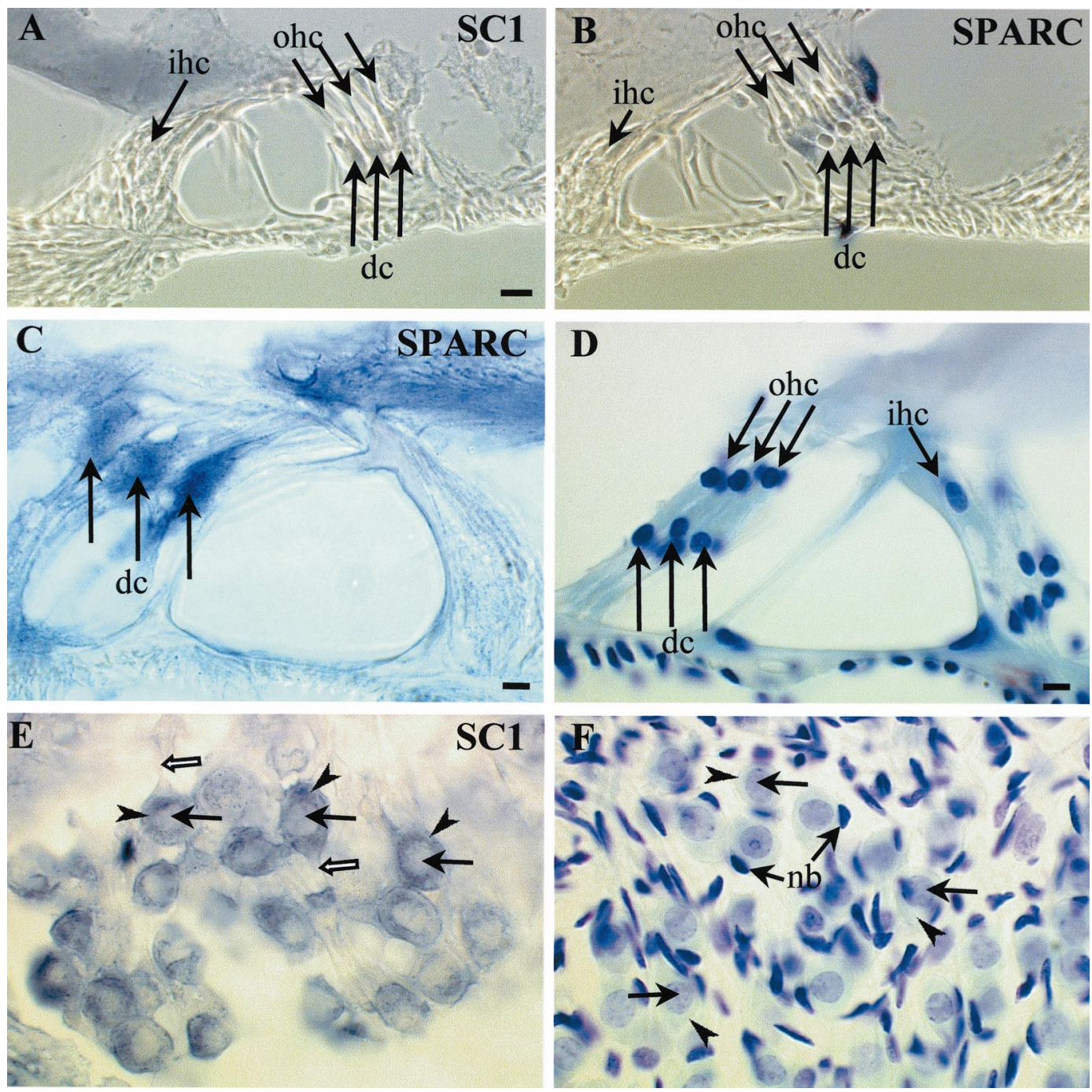

Fig. 5. SPARC mRNA is expressed in dc of the oc at P10. (A) and (B) are high magnification phase-contrast views of the oc at P10 following non-radioactive in situ hybridization with SC1 and SPARC antisense riboprobes, respectively. SC1 mRNA expression is not apparent in the oc (A). However, a striking induction of SPARC mRNA is localized to the elongated dc, but not the sensory cells, such as the ihc and ohe whose base is supported by the dc (B and C). (D) is a section that is stained with cresyl violet to show cellular morphology and structure of the oc at high magnification. An adjacent section indicates that SPARC transcripts are localized to the apical cup region of the dc in the oc (C). High magnification views of the sg of P20 cochleas stained with cresyl violet show neurons with large darkly staining nuclei (arrows), paler cytoplasm (arrowheads), and darkly staining nb characteristic of type I sgn (F). (E) shows an adjacent section hybridized with DIG-labeled SCl probes indicating a cluster of type I sgn strongly expressing SC1 mRNA. Signal is enriched in the cytoplasm of the cell body of these sensory neurons (arrowheads) and does not appear to extend into cellular processes (open arrows). Scale bar $=17.3 \mu \mathrm{m}$ for $(\mathrm{A})$ and $(\mathrm{B})$; bar $=10.9 \mu \mathrm{m}$ for $(\mathrm{C})-$ (F). dc, Deiters cells; ihc, inner hair cells; nb, Nissl body; ohc, outer hair cells; arrows, nuclei; open arrows, processes; arrowheads, cytoplasm.

regions (Fig. 6A). The high levels of SC1 mRNA observed in sgn at earlier ages was maintained through to P14 (Fig. 6C,E) and adult (data not shown). As seen at P20 in the sg, SC1 mRNA was enriched in the cell body cytoplasm (arrowheads in Fig. 5E) surrounding the large nuclei characteristic of type I sgn (indicated by arrows in Fig. 5E and in the adjacent cresyl violet stained section in Fig. 5F). Type I sgn constitute the majority of cells within the ganglion. These neurons are characterized by their large cell body and round nuclei, and an abundance of Nissl bodies (nb) in the cytoplasm (rough endoplasmic reticulum) that stain darkly as seen in Fig. 5F (Schwartz, 1986). SC1 mRNA did not appear to extend out into the cellular processes of these sensory neurons (processes indicated by open arrows in Fig. 5E). In comparison, SPARC mRNA was not detected in sgn from E20 (Fig. 1) through to P14 (Fig. 6D,F), and adult (not shown).

At P14, high levels of SPARC mRNA continued to be associated with the oc, sl (Fig. 6D,F) and type I fibrocytes of the slg (Fig. 6D,F,H). In contrast, SC1 mRNA was not detected in the oc and sl, and the dis- 

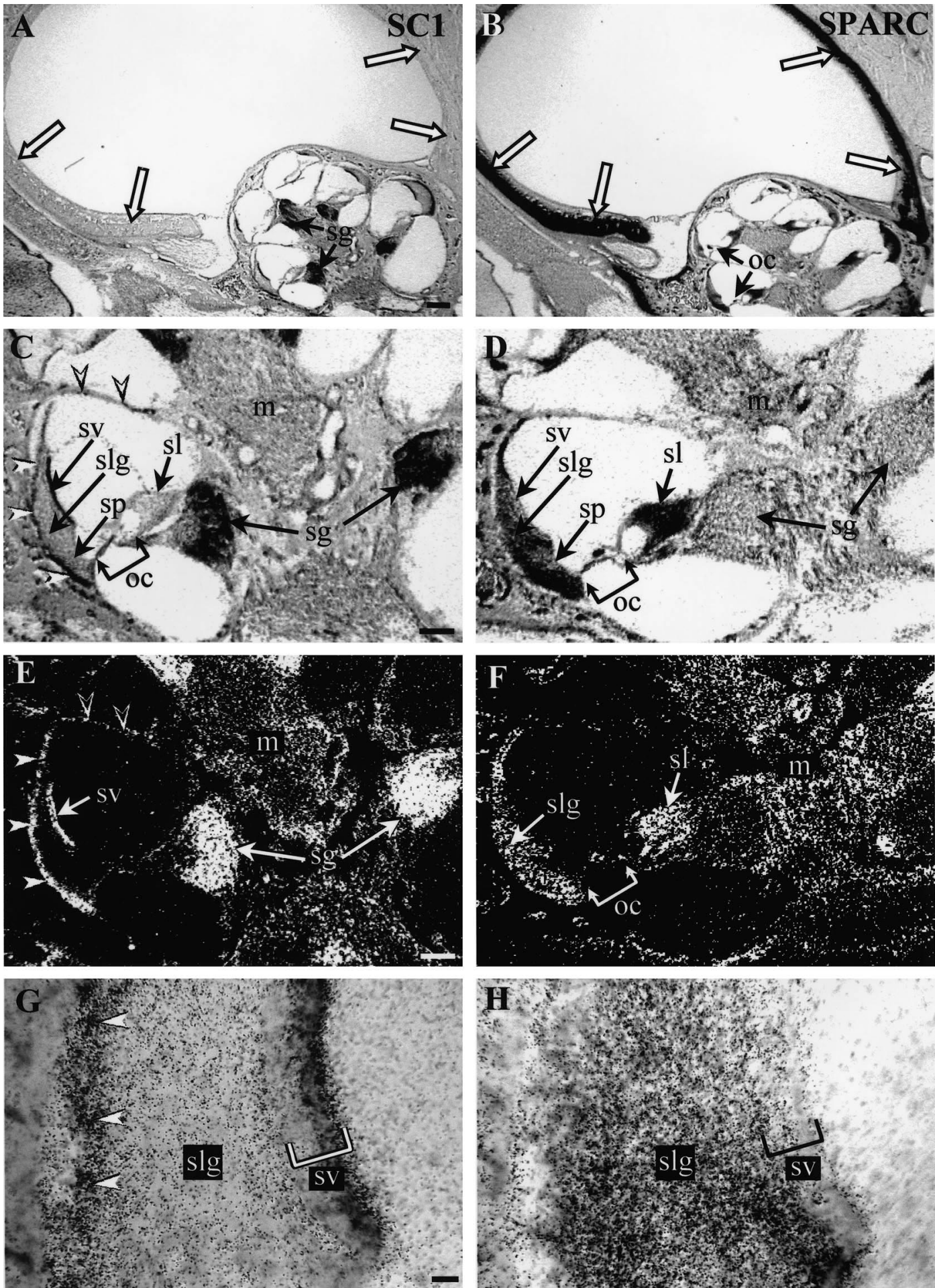

Fig. 6. Continued expression of SPARC mRNA in the oc at P14. (A) and (B) are low magnification views of the cochlea at P14 showing strong SPARC mRNA expression in the temporal bone (open arrows) and oc, regions where SC1 mRNA is not expressed. SPARC mRNA continues to be expressed at high levels in the sl, slg, and in the supporting dc of the oc (D and F). (G) and (H) are high magnification views of the cochlear lateral wall. SPARC transcripts are localized to type I fibrocytes of the $\operatorname{slg}(\mathrm{H})$, whereas type III fibrocytes of the slg (white arrowheads in $\mathrm{C}, \mathrm{E}$, and $\mathrm{G}$ ) and marginal cells of the sv strongly express SC1 mRNA (G). Neither transcript is expressed by type II fibrocytes of the sp (C and D). Scale bar $=450 \mu \mathrm{m}$ for $(\mathrm{A})$ and $(\mathrm{B})$; bar $=220 \mu \mathrm{m}$ for $(\mathrm{C})-(\mathrm{F})$; bar $=17.3 \mu \mathrm{m}$ for $(\mathrm{G})$ and $(\mathrm{H})$. $\mathrm{m}$, modiolus; oc, organ of Corti; sg, spiral ganglion; sl, spiral limbus; slg, spiral ligament; sp, spiral prominence; sv, stria vascularis; open arrowheads, lining of scala compartments; white arrowheads, type III fibrocytes of slg; open arrows, temporal bone. 
tribution of SC1 transcripts was highly localized in the lateral wall of the cochlea (Fig. 6C,E). The intense SC1 mRNA signal was localized to the marginal cells of the sv which are epithelial cells that line the luminal surface of the sv (Fig. 6G), and type III fibrocytes of the slg (indicated by the white arrowheads in Fig. 6C,E,G). It was apparent that lower levels of SC1 mRNA were expressed by type I fibrocytes of the slg at P14 than at P10 (compare Fig. 6E,G and Fig. 4C,E). As reflected at P10 (Fig. 4C-F), at P14 neither SC1 nor SPARC transcripts appear to be expressed by type II fibrocytes of the sp (Fig. 6C,D). In the P14 oc, SPARC mRNA was localized to the region of dc (Fig. 6D,F), as was seen at P10 (Fig. 5B,D). The SC1 and SPARC mRNA expression patterns which were observed at P14 were maintained in the adult (data not shown).

\section{Discussion}

Recently there has been much interest in the characterization of cochlea-specific genes in order to facilitate the identification of candidate genes for human hearing disorders and to increase the understanding of auditory function at the molecular level. Sequence analysis of randomly chosen clones from human fetal inner ear or cochlear cDNA libraries has revealed an abundance of signal transduction proteins, trafficking proteins and components of the ECM (Robertson et al., 1994; Jacob et al., 1997; Skvorak et al., 1999). A number of ECM genes are expressed in the inner ear, serving structural or regulatory functions. It is apparent that defects in many of these genes such as type IV collagens, $\alpha$-tectorin, Usher IIA protein, collagen 11A2, result in inherited forms of hearing impairment (Petit, 1996; Corwin, 1998; Mustapha et al., 1999; Cremers, 1999; McGuirt et al., 1999).

Interestingly, SPARC is one of the most highly expressed genes in the human fetal cochlea (Robertson et al., 1994; Jacob et al., 1997; Skvorak et al., 1999). SPARC is expressed in tissues undergoing reorganization and is thought to modulate cell-matrix interactions by inhibiting cell spreading and cell cycle progression and by regulating the production of other ECM molecules (Motamed, 1999). SPARC and the related SC1 are secreted glycoproteins that share similar motifs such as a repeated domain in follistatin, high-affinity calcium-binding sites and the ability to bind other ECMs (Mason et al., 1986; Johnston et al., 1990; Girard and Springer, 1995). SPARC and hevin, the human homolog of rat $\mathrm{SC1}$, possess anti-adhesive properties which lead to cell rounding and changes in cell shape (Sage et al., 1989; Girard and Springer, 1996) which are necessary for cells undergoing migration and proliferation. Hevin was also identified from ESTs generated and sequenced from a human cochlear library (Skvorak et al., 1999). Consequently, we were interested in determining the mRNA expression patterns of SPARC and $\mathrm{SC} 1$ in the developing rat cochlea. Unlike humans, rats are unable to hear at birth and their auditory function develops during the second and third postnatal weeks (Uziel et al., 1981; Rybak et al., 1992). This postnatal maturation feature of the rat auditory system allows the investigation of gene expression patterns prior to and following the onset of hearing.

The first two postnatal weeks is a critical period for inner ear development in the rat. It is during this period that many major morphological characteristics of the $\mathrm{oc}$, sg, and sv are established. For example, neurons innervate the oc, the cochlear endolymph and endocochlear potential are established, and the cochlea becomes functional (Uziel et al., 1981; Schwartz, 1986; Lim and Rueda, 1992; Rybak et al., 1992; Sobkowitz, 1992). The cochlea consists of a spectrum of structurally and functionally different cell types. The slg, sl, and $\mathrm{m}$ are connective tissue elements of the membranous cochlea, while hair cells of the oc and the sg constitute neuronal elements. In the oc, the hair cells and supporting cells are difficult to distinguish in the newborn rat since the receptor cells and supporting cells form a compact epithelial lining on the basilar membrane. Up to 8 days after birth, few morphological changes occur. Between P8 and P12, the extracellular fluid spaces have formed and the supporting cells have increased remarkably in length, reflecting the mature morphology of the oc (Roth and Bruns, 1992).

Comparison of in situ signal on sections obtained from decalcified and non-decalcified specimens revealed no change in expression pattern or strength of signal. Previous studies have shown that decalcification has no detectable effect on mRNA hybridization (Ryan et al., 1991). Representative sections of decalcified tissue processed for in situ hybridization were presented since this allowed for better tissue morphology.

At the earliest stage of cochlear development that was examined, E20, SC1 mRNA was expressed at high levels in sgn. The sg is composed of a spiral shaped mass of nerve cell bodies of bipolar sensory neurons which relay information from the hair cells to the cochlear nucleus in the brainstem (Schwartz, 1986). Throughout development and in the adult, an intense SC1 mRNA signal was observed in large type I neurons which comprise the majority of cell types within the sg. This suggests that $\mathrm{SC1}$ may be important for the maintenance of sgn function. It is interesting that SC1 mRNA is also expressed in ganglion neurons of the retina throughout development and in the adult (Mothe and Brown, 2000). The retinal ganglion cells serve a similar role in that these neurons are the primary sensory neurons relaying visual information from the pho- 
toreceptors to the brain. This suggests that SC1 could play a common fundamental role in these primary sensory neurons. In both the retinal ganglion neurons and cochlear neurons of the sg, SC1 mRNA was distributed throughout the cytoplasm of the cell bodies of these neurons at all stages of development but not into neuronal processes. We have previously shown that $\mathrm{SCl}$ mRNA is selectively transported into cellular processes of Bergmann glial cells of the cerebellum at particular stages of development (Mothe and Brown, 2000). In contrast, SPARC expression was not apparent in the sg during development or in the adult. SPARC mRNA was enriched in the cartilaginous and ossifying otic capsule surrounding the cochlea and in the temporal bone. SC1 mRNA was not apparent in these regions. Previous studies have shown that SPARC is one of the most abundant non-collagenous proteins in cartilage and bone (Lane and Sage, 1994).

At birth, the posterior wall of the cochlear duct is organized into the greater and lesser epithelial ridge which harbors the developing oc. The tall columnar cells of the epithelial ridge recede and undergo apoptosis, and as a result, the sl and inner sulcus is formed by about P7 in the rat (Lim and Rueda, 1992). This process involves cell differentiation and migration, as well as cell death and resorption. SPARC and particularly SC1 mRNA, exhibit an intense signal in the ger between E20 and P5. Therefore, the expression of these related anti-adhesive ECMs could play a role in the remodeling of the developing oc, mediating cell migration and changes in cell shape.

The intense SC1 mRNA signal apparent in the ger at E20, P1 and P5 may also reflect the synthesis of SC1 by these cells and the subsequent secretion of the encoded ECM protein at the critical time in development when the tectorial membrane is forming. The mammalian tectorial membrane is an acellular ECM-rich flap in which the hair cells and their stereocilia are embedded (Kuijpers and Manni, 1986). The tectorial membrane is an essential component involved in the transduction of auditory information. This acellular structure is comprised of collagens and glycoconjugates such as proteoglycans and glycoproteins (Lim and Rueda, 1992). Since the tectorial membrane is an acellular structure and thus does not make its own proteins, a number of investigations have focused on determining the source of the ECM proteins comprising the tectorial membrane. Studies have shown that collagens, glycoproteins and proteoglycans are synthesized in the ger and subsequently secreted to the tectorial membrane (Kuijpers and Manni, 1986; Khetarpal et al., 1994; Khetarpal and Morton, 1998; Rau et al., 1999). Given the specific localization of SC1 mRNA in the ger, particularly to the apical region, it is conceivable that $\mathrm{SCl}$ may be synthesized in these cells and then secreted and incor- porated in the ECM comprising the tectorial membrane.

Throughout cochlear development, both $\mathrm{SCl}$ and SPARC mRNA are expressed in connective tissues involved in ion homeostasis, such as the epithelial lining of the scala compartments containing the perilymphatic fluid. However, these transcripts are differentially localized to connective tissue elements of the slg and sv which are responsible for generating the endocochlear potential which is critical for auditory function. For example, at P1, higher levels of SC1 mRNA are associated with the developing sv as compared to adjacent fibrocytes of the slg, while the reverse pattern is observed for SPARC. This differential expression of transcripts is even more pronounced at P10 and P14 in that SC1 mRNA is enriched in marginal cells of the sv and type III fibrocytes of the slg. In contrast, SPARC expression is higher in type I fibrocytes of the slg than the sv. Both the sv and slg are important in the maintenance of homeostasis of the inner ear fluids. It has been suggested that these structures play a role in ion and fluid transport since they contain high concentrations of ion transport enzymes, such as Na, K-ATPase and carbonic anhydrase (Spicer and Schulte, 1991).

In the slg, fibrocytes are classified according to their location and structural and biochemical differences (Spicer and Schulte, 1991, 1996). For example, type I fibrocytes, which strongly express SPARC mRNA, occupy the area between the sv and the otic capsule. In contrast, $\mathrm{SC} 1 \mathrm{mRNA}$ is most strongly expressed in the type III fibrocytes which line the otic capsule. However, neither transcript appears to be expressed by type II fibrocytes which are found in the sp. These fibrocytes all communicate with each other via gap junctions (Spicer and Schulte, 1996). A strong distribution of SPARC transcripts is evident in the sl at P10 and maintained in the adult. Like the slg, the sl is composed of fibrocytes which are interconnected by gap junctions and derived from the surrounding mesoderm. This cochlear structure is also postulated to play a role in ion homeostasis (Kikuchi et al., 1995).

In the sv, SC1 mRNA appears to be localized to the secretory marginal epithelial cells that line the luminal surface of this structure. This is seen at P14 when the mature morphology of the sv is evident. In the mature cochlea, the sv is composed of three cell layers: (1) an outer layer of marginal cells bordering the endolymphatic space, (2) an intermediate cell layer and (3) an inner most layer of basal cells. It is interesting that $\mathrm{SC1}$ mRNA is specifically localized to the marginal cells of the sv and cells of the ger, both of which are secretory cell types. The localization of SC1 and SPARC mRNA to cochlear structures that contain an abundance of ion transport enzymes suggest a homeostatic role for these ECM molecules perhaps involving calcium ion regula- 
tion. Similarly, osteopontin expression is coincident in these cochlear tissues which contain an abundance of ion transport enzymes (Lopez et al., 1995). Osteopontin is a secreted, sialic acid-rich protein that binds calcium ions with low affinity and interacts with cells through integrin receptors. In addition, this ECM protein is a major constituent of cochlear fluids, and it has been suggested that the marginal cells of the sv produce and secrete osteopontin into the endolymph (Lopez et al., 1995).

At P10, a dramatic change in SPARC expression occurred. SPARC mRNA was induced in the oc, in the supporting dc. This onset of mRNA expression is particularly interesting since this timepoint correlates with the onset of hearing in rats which occurs between P9 and P11 (Uziel et al., 1981; Rybak et al., 1992). The cellular mosaic of the mammalian oc represents one of the most highly ordered structures in any vertebrate system (Raz and Kelley, 1999). The oc consists of inner hair cells (ihc) and ohc surrounded by various types of supporting cells which are separated by fluid-filled spaces. Ihc serve a mainly sensory function in relaying information to the sgn (Spoendlin, 1986), while ohc respond to mechanical stimuli by generating receptor potentials (Dallos et al., 1982). Supporting cells, such as the de lie below the sensory cells and appear to provide the mechanical support required to hold the sensory cells up off the basilar membrane and to transmit movement of the basilar membrane to the stereocilia on the hair cells (Pack and Slepecky, 1995). These $\mathrm{dc}$ are closely apposed to the ohc, extending a phalangeal process apically which surrounds the ohc and contributes to the reticular lamina. Thus, these cells are thought to serve an important role as a trophic intermediary for the ohc, contributing towards their metabolism.

Interestingly, the localization of SPARC mRNA to dc correlates with SPARC expression in the slg. These cell types are involved in maintaining ionic homeostasis. Potassium that leaks from the scala media through the oc is readsorbed by dc, and diffuses through supporting cells via gap junctions, and is then directed to the sv through slg fibrocytes (Lecain et al., 1999). SPARC may be involved in maintaining homeostasis, perhaps in a calcium-buffering capacity. This ECM molecule can bind calcium with both high and low affinity (Maurer et al., 1992; Pottgiesser et al., 1994). It has been suggested that ohc have efficient calcium-buffering mechanisms to maintain low levels of calcium (Slepecky and Ulfendahl, 1993). A number of calcium-binding proteins have been shown to be expressed in supporting cells such as dc and sensory hair cells (Dechesne et al., 1993; Slepecky and Ulfendahl, 1993; Pack and Slepecky, 1995; Sakaguchi et al., 1998). Calcium is thought to play a major signaling role in ohc to regulate cytoskeletal integrity, cell shape and cell excitability (Slepecky and Ulfendahl, 1993). Given the close association of ohe to surrounding dc which function as trophic intermediaries, the expression of SPARC in dc may be important in calcium regulation. At the onset of hearing, the need for rapid changes in calcium levels in hair cells is even more apparent. Therefore, the induction of SPARC mRNA in dc during the onset of hearing and the maintained expression through to the adult suggests that this ECM molecule may function to regulate or buffer calcium levels.

Recently, mice deficient for SPARC have been generated and they appear to develop normally except for the formation of early-onset cataracts (Gilmour et al., 1998; Norose et al., 1998; Bassuk et al., 1999). Similarly, SC1 knockouts have been reported to show no obvious deficits (McKinnon et al., 2000). Given the present results of the expression of SPARC and SC1 mRNA in the cochlea, it would be interesting to determine if there are any auditory deficits associated with these knockout models, and if there is any morphological perturbation of cochlear structures.

\section{Acknowledgements}

This work was supported by NSERC Canada. We are grateful to Sheila Rush for critical reading of this manuscript.

\section{References}

Bassuk, J.A., Birkebak, T., Rothmier, J.D., Clark, J.M., Bradshaw, A., Muchowski, P.J., Howe, C.C., Clark, J.I., Sage, E.H., 1999. Disruption of the SPARC locus in mice alters the differentiation of lenticular epithelial cells and leads to cataract formation. Exp. Eye Res. 68, 321-331.

Corwin, J.T., 1998. Identifying the genes of hearing, deafness, and disequilibrium. Proc. Natl. Acad. Sci. USA 95, 12080-12082.

Cosgrove, D., Rodgers, K.D., 1997. Expression of the major basement membrane-associated proteins during postnatal development in the murine cochlea. Hear. Res. 105, 159-170.

Cremers, F.P.M., 1999. Genetic causes of hearing loss. Curr. Opin. Neurol. 11, 11-16.

Dallos, P., Santos-Sacchi, J., Flock, A., 1982. Intracellular recording from cochlear outer hair cells. Science 218, 582-584.

Dechesne, C.J., Winsky, L., Moniot, B., Raymond, J., 1993. Localization of calretinin mRNA in rat and guinea pig inner ear by in situ hybridization using radioactive and non-radioactive probes. Hear. Res. 69, 91-97.

Gilmour, D.T., Lyon, G.J., Carlton, M.B., Sanes, J.R., Cunningham, J.M., Anderson, J.R., Hogan, B.L., Evans, M.J., Colledge, W.H., 1998. Mice deficient for the secreted glycoprotein SPARC/osteonectin/BM40 develop normally but show severe age-onset cataract formation and disruption of the lens. EMBO J. 17, 1860-1870.

Girard, J.P., Springer, T.A., 1995. Cloning from purified high endothelial venule cells of hevin, a close relative of the anti-adhesive extracellular matrix protein SPARC. Immunity 2, 113-123. 
Girard, J.P., Springer, T.A., 1996. Modulation of endothelial cells adhesion by hevin, an acidic protein associated with high endothelial venules. J. Biol. Chem. 271, 4511-4517.

Holland, P.W.H., Harper, S.J., McVey, J.H., Hogan, B.L.M., 1987. In vivo expression of mRNA for the calcium-binding protein SPARC (osteonectin) revealed by in situ hybridization. J. Cell Biol. 105, 473-482.

Jacob, A.N.K., Baskaran, N., Kandpal, G., Narayan, D., Bhargava, A.K., Kandpal, R.P., 1997. Isolation of human ear specific cDNAs and construction of cDNA libraries from surgically removed small amounts of inner ear tissues. Somat. Cell Mol. Genet. 23, 83-95.

Johnston, I.G., Paladino, T., Gurd, J.W., Brown, I.R., 1990. Molecular cloning of $\mathrm{SC1}$ : a putative brain extracellular matrix glycoprotein showing partial similarity to osteonectin/BM40/SPARC. Neuron 2, 165-176

Khetarpal, U., Robertson, N.G., Yoo, T.J., Morton, C.C., 1994. Expression and localization of COL2A1 mRNA and type II collagen in human fetal cochlea. Hear. Res. 79, 59-73.

Khetarpal, U., Morton, C.C., 1998. Inner ridge cells may be the main source of tectorial membrane type II collagen: evidence from quantitative mRNA in situ hybridization. Acta Otolaryngol. 118, 177-184.

Kikuchi, T., Kimura, R.S., Paul, D.S., Adams, J.C., 1995. Gap junctions in the rat cochlea: immunohistochemical and ultrastructural analysis. Anat. Embryol. 191, 101-118.

Kuijpers, W., Manni, J.J., 1986. Developmental aspects of glycoprotein secretion and migration in the endolymphatic space. Acta Otolaryngol. S429, 35-43.

Lane, T.F., Sage, E.H., 1994. Review: the biology of SPARC a protein that modulates cell-matrix interactions. FASEB J. 8, 163-173.

Lecain, E., Sauvaget, E., Crisanti, P., Van Den Abbeele, T., Tran Ba Huy, P., 1999. Potassium channel ether a go-go mRNA expression in the spiral ligament of the rat. Hear. Res. 133, 133-138.

Legan, P.K., Richardson, G.P., 1997. Extracellular matrix and cell adhesion molecules in the developing inner ear. Semin. Cell Dev. Biol. 8, 217-224.

Lim, D.J., Rueda, J., 1992. Structural development of the cochlea. In: Romand, R. (Ed.), Development of Auditory and Vestibular Systems 2. Elsevier, Amsterdam, pp. 33-58.

Lopez, C.A., Olson, E.s., Adams, J.C., Mous, K., Denhardt, D.T., Davis, R.L., 1995. Osteopontin expression detected in adult cochleae and inner ear fluids. Hear. Res. 85, 210-222.

Mason, I.J., Taylor, A., Williams, J.G., Sage, H., Hogan, B.L., 1986. Evidence from molecular cloning that SPARC, a major product of mouse embryo parietal endoderm, is related to an endothelial 'culture shock' glycoprotein. EMBO J. 5, 1465-1472.

Maurer, P., Mayer, U., Bruch, M., Jeno, P., Mann, K., Landwehr, R., Engel, J., Timpl, R., 1992. High-affinity and low-affinity calcium binding and stability of the multidomain extracellular $40-\mathrm{kDa}$ basement membrane glycoprotein (BM-40/SPARC/osteonectin). Eur. J. Biochem. 205, 233-240.

McGuirt, W.T., Prasad, S.D., Griffith, A.J., Kunst, H.P.M., Green, G.E., Shpargel, K.B., Runge, C., Huybrechts, C., Mueller, R.F., Lynch, E., King, M.-C., Brunner, H.G., Cremers, W.R.J., Takanosu, M., Li, S.-W., Arita, M., Mayne, R., Prockop, D., Van Camp, G., Smith, R.J.H., 1999. Mutations in COL11A2 cause non-syndromic hearing loss (DFNA13). Nat. Genet. 23, 413-419.

McKinnon, P.J., McLaughlin, S.K., Kapsetaki, M., Margolskee, R.F., 2000. Extracellular matrix-associated protein SC1 is not essential for mouse development. Mol. Cell Biol. 20, 656-660.

Motamed, K., 1999. Review: SPARC (osteonectin/BM-40). Int. J. Biochem. Cell Biol. 31, 1363-1366.

Mothe, A.J., Brown, I.R., 2000. Selective transport of SC1 mRNA, encoding a putative extracellular matrix glycoprotein, during post- natal development of the rat cerebellum and retina. Mol. Brain Res. 76, 73-84.

Mustapha, M., Weil, D., Chardenoux, S., Elias, S., El-Zir, E., Beckmann, J.S., Loiselet, J., Petit, C., 1999. An $\alpha$-tectorin gene defect causes a newly identified autosomal recessive form of sensorineural pre-lingual non-syndromic deafness, DFNB21. Hum. Mol. Genet. $8,409-412$

Norose, K., Clark, J.I., Syed, N.A., Basu, A., Heber-Katz, E., Sage, E.H., Howe, C.C., 1998. SPARC deficiency leads to early-onset cataractogenesis. Invest. Ophthalmol. Vis. Sci. 39, 2674-2680.

Pack, A.K., Slepecky, N.B., 1995. Cytoskeletal and calcium-binding proteins in the mammalian organ of Corti: cell type-specific proteins displaying longitudinal and radial gradients. Hear. Res. 91, $119-135$.

Petit, C., 1996. Genes responsible for human hereditary deafness: symphony of a thousand. Nat. Genet. 14, 385-391.

Pottgiesser, J., Maurer, P., Mayer, U., Nischt, R., Mann, K., Timpl, R., Krieg, T., Engel, J., 1994. Changes in calcium and collagen IV binding caused by mutation in the EF hand and other domains of extracellular matrix protein BM-40 (SPARC, osteonectin). J. Mol. Biol. 238, 563-574.

Rau, A., Legan, P.K., Richardson, G.P., 1999. Tectorin mRNA expression is spatially and temporally restricted during mouse inner ear development. J. Comp. Neurol. 405, 271-280.

Raz, Y., Kelley, M.W., 1999. Retinoic acid signaling is necessary for the development of the organ of Corti. Dev. Biol. 213, 180-193.

Robertson, N.G., Khetarpal, U., Gutierrez-Espeleta, G.A., Bieber, F.R., Morton, C.C., 1994. Isolation of novel and known genes from a human fetal cochlear cDNA library using subtractive hybridization and differential screening. Genomics 23, 42-50.

Roth, B., Bruns, V., 1992. Postnatal development of the rat organ of Corti II. Hair cell receptors and their supporting elements. Anat. Embryol. 185, 571-581.

Ryan, A.F., Watts, A.G., Simmons, D.M., 1991. Preservation of mRNA during in situ hybridization in the cochlea. Hear. Res. $56,148-152$.

Rybak, P.L., Whitworth, C., Scott, V., 1992. Development of endocochlear potential and compound action potential in the rat. Hear. Res. 59, 189-194.

Sage, E.H., Vernon, R.B., Funk, S.E., Everitt, E.A., Angello, J., 1989. SPARC, a secreted protein associated with cellular proliferation, inhibits cell spreading in vitro and exhibits $\mathrm{Ca}^{+2}$-dependent binding to the extracellular matrix. J. Cell Biol. 109, 341-356.

Sakaguchi, N., Henzl, M.T., Thalmann, I., Thalmann, R., Schulte, B.A., 1998. Oncomodulin is expressed exclusively by outer hair cells in the organ of Corti. J. Histochem. Cytochem. 46, 2939.

Schwartz, A.M., 1986. Auditory nerve and sprial ganglion cells: morphology and organization. In: Altschuler, R.A. et al. (Eds.), Neurobiology of Hearing: the Cochlea. Raven Press, New York, pp. 271-282.

Skvorak, A.B., Weng, Z., Yee, A.J., Robertson, N.G., Morton, C.C., 1999. Human cochlear expressed sequence tags provide insight into cochlear gene expression and identify candidate genes for deafness. Hum. Mol. Genet. 8, 439-452.

Slepecky, N.B., Ulfendahl, M., 1993. Evidence for calcium-binding proteins and calcium-dependent regulatory proteins in sensory cells of the organ of Corti. Hear. Res. 70, 73-84.

Sobkowitz, H.M., 1992. The development of innervation in the Organ of Corti. In: Romand, R. (Ed.), Development of Auditory and Vestibular Systems 2. Elsevier, Amsterdam, pp. 59-100.

Spicer, S.S., Schulte, B.A., 1991. Differentiation of inner ear fibrocytes according to their ion transport related activity. Hear. Res. 56, 5364.

Spicer, S.S., Schulte, B.A., 1996. The fine structure of spiral ligament 
cells relates to ion return to the stria and varies with place-frequency. Hear. Res. 100, 80-100.

Spoendlin, H., 1986. Receptoneural and innervation aspects of the inner ear anatomy with respect to cochlear mechanics. Scand. Audiol. 25 ((Suppl.)), 27-34.

Steel, K.P., Bussoli, T.J., 1999. Deafness genes, expression of surprise. Trends Genet. 15, 207-211.

Uziel, A., Romand, R., Marot, M., 1981. Development of cochlear potentials in rats. Audiology 20, 89-100.

Whitlon, D.S., Zhang, X., Kusakabe, M., 1999. Tenascin-C in the cochlea of the developing mouse. J. Comp. Neurol. 406, 361374.

Woolf, N.K., Koehrn, F.J., Ryan, A.F., 1992. Immunohistochemical localization of fibronectin-like protein in the inner ear of the developing gerbil and rat. Dev. Brain Res. 65, 21-23.

Yan, Q., Sage, E.H., 1999. Review: SPARC, a matricellular glycoprotein with important biological functions. J. Histochem. Cytochem. 47, 1495-1505. 